\title{
Oncoplastic Breast Surgery in Gynecologic Oncology Breast Pole to the National Institute of Rabat
}

\author{
J. A. M'bongo*, Y. El Alami, W. L. Tatsipie Meukem, F. Tijami, Z. Hanchi, H. Hachi \\ Gynecomammary Surgery Department, Rabat, Morocco \\ Email: *mbongojalf@gmail.com
}

How to cite this paper: M'bongo, J.A., El Alami, Y., Meukem, W.L.T., Tijami, F., Hanchi, Z. and Hachi, H. (2019) Oncoplastic Breast Surgery in Gynecologic Oncology Breast Pole to the National Institute of Rabat. Open Journal of Obstetrics and $G y$ necology, 9, 997-1004.

https://doi.org/10.4236/ojog.2019.97097

Received: May 10, 2019

Accepted: July 19, 2019

Published: July 22, 2019

Copyright $\odot 2019$ by author(s) and Scientific Research Publishing Inc. This work is licensed under the Creative Commons Attribution International License (CC BY 4.0).

http://creativecommons.org/licenses/by/4.0/

c. (i) Open Access

\begin{abstract}
Goal: Evaluate the techniques and results obtained from the mammary Oncoplastic in the conservative treatment of breast cancer. Patients and methods: This is a retrolective descriptive study conducted during the period from 3 April 2017 to 3 April 2019, the Gynecologic Breast pole of the Oncology National Institute of Rabat. Results: Out of 105 files listed, the locations of the tumours were: $35 \% 37 / 105$ QSE, QSI QSE + 21/105 or 20\%, QSI 19/105 or $18 \%$, QSI $12 / 105$ or $11 \%$, other maps $16 / 105$ or $16 \%$. The techniques were Oncoplastic: Pamectomy $10 / 105$ or $10 \%$ Round Block $58 / 105$ or $55 \%$, plasty inverted T $21 / 105$ or $20 \%$, and other technical $16 / 105$ or $15 \%$. The aesthetic results and the quality of resection were correct and above all stable at 82/105 patients $78 \%$ of cases. The lymphocele $13 / 105$ or $12 \% 5 / 1055 \%$ lymphoedema represented early complications occurred as late complications consisted of retractile unsightly scars $15 / 105$ or $14 \%$, breast asymmetry $13 / 105$ soit $12 \%$ and cutaneous sclerosis $3 / 105$ or $3 \%$. Conclusion: The oncoplastic surgery is a part of multidisciplinary management of breast cancer. It imposes oncologic resections and reassuring cosmetic results for the patient and the practitioner.
\end{abstract}

\section{Keywords}

Breast Oncoplasty, Rabat-Morocco

\section{Introduction}

Breast cancer is a public health problem. If the type Patey mastectomy causes impairment of body image and femininity, conservative treatment of breast cancer becomes the reference to respect the integrity of the breast and the body of the patient. Oncoplastic surgery is the use of plastic surgery techniques at the 
conservative treatment of breast cancer. The tumor resection correlates with a plastic gesture of glandular defect filling to preserve the morphology of the breast and improve cosmetic results [1]. Currently, the trend is the expansion of indications of conservative treatment because of major advances in the early detection of subclinical lesions and providing treatment neoadjuvant chemotherapy [2] or radiotherapy first [3]. Conservative breast cancer treatment is currently the standard treatment, whether in palpable tumours or intra-clinical lesions. Oncoplastic surgery plays an important role in the choice of surgical treatments and reduces the number of mastectomies. Thus, it seemed appropriate for us to evaluate the techniques and results obtained from breast oncoplasty as part of the conservative treatment of breast cancer in our department.

\section{Patients and Methods}

This is a descriptive study with retrolection collection of data held in the breast oncology Gynecological Surgery Oncology at the National Institute of Rabat during the period from 3 January 2017 to 3 April 2019. For example, some patients were followed for two years while others were followed for only two months.

Included were the records of patients with a breast size of less than 5 centimetres, where the shape of the breast suggests the best oncological and esthetic outcome and a degree of ptosis compatible with oncoplasty and having performed radiotherapy.

Incomplete files and files with locally advanced tumors, two tumors located in separate quadrants of the breast, micro calcifications occupying more than a quarter of the breast volume, patients' choices in favor of mastectomy were excluded.

\section{Results}

We identified 105 patients records have submitted T1 and T2 breast cancer who underwent conservative surgery coupled with Oncoplastic according to tumor location.

The average age of patients 47 years (range 34 to 68 years), 90 patients without occupation $86 \%, 15$ had a gainful occupation $14 \%$.

The tumor was localized to the supero-external quadrant in half of the cases (Figure 1).

The Oncoplastic techniques were used depending on the location of the tumor.

Round Block has been practiced in more than half the cases (Figure 2).

The aesthetic results and the quality of resection were correct (excellent, good or fairly good) and especially stable at $82 / 105$ patientes $78 \%$ of the cases ( 8 and 9 ).

We have listed the following complications: the asymmetric position of the areola $(12 / 105) 12 \%$ of cases; breast deformity by loss of glandular substance (16/105) 15\%; skin retraction (15/105) 14\%, the widened scar, retractable and 


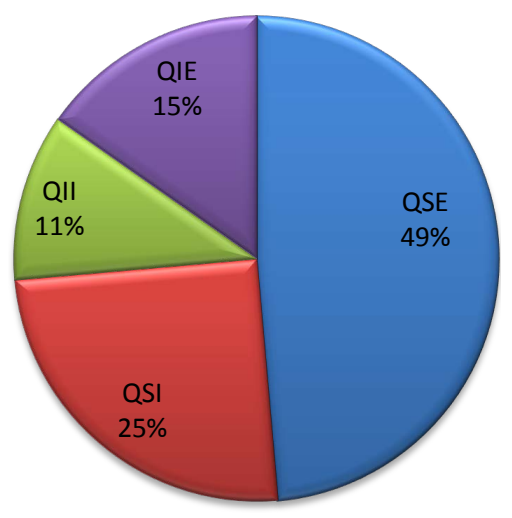

Figure 1. Locations of the tumor. QIE: infero-outer quadrant; QII: intero inner quadrant; QSE: upper outer quadrant; QSI: upper-inner quadrant.
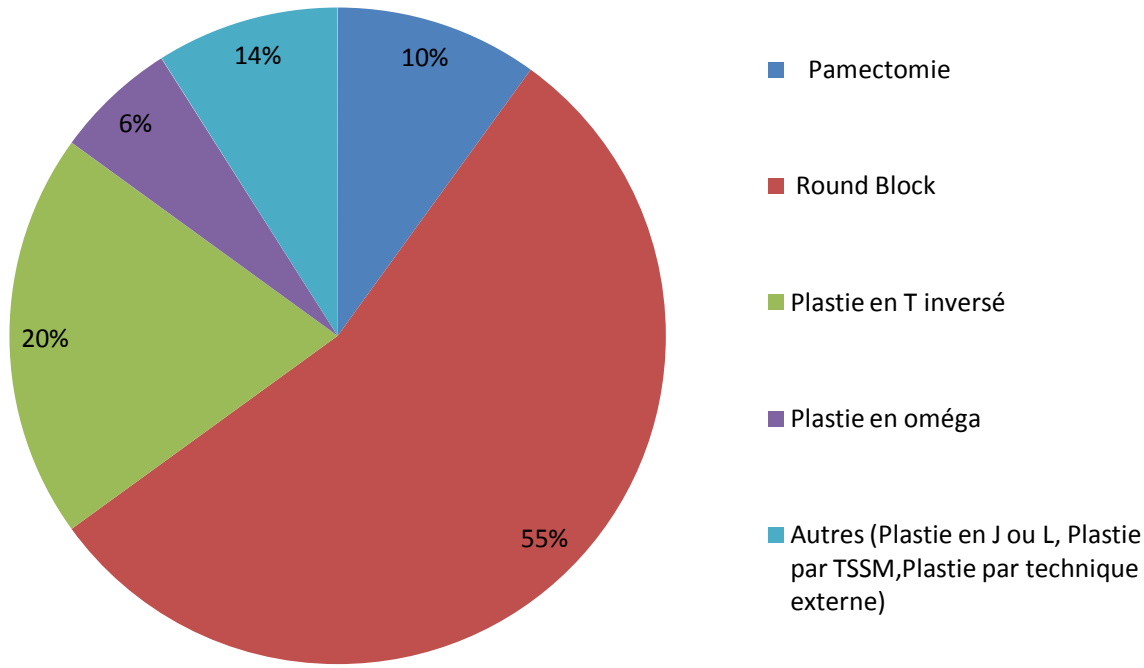

Figure 2. Oncoplastic techniques.

unsightly or Scars flange (3/105) 3\%. The cosmetic results were difficult to treat and surgical times between $5 \%$ to $10 \%$.

\section{Comments}

Oncoplastic surgery is the use of plastic surgery techniques in the conservative treatment of breast cancer. The tumor resection correlates with a plastic gesture of glandular defect filling to preserve the breast morphology and improve cosmetic results [4].

Oncoplastic surgery plays a full role in the choice of surgical treatment of breast cancer, whether palpable tumors or infra radiological clinical lesions. It reduces the number of mastectomy, and get greater local control of breast cancer, getting through resections off sufficient safety margins. Surgical options should be developed in a precise and consensual manner according to the patient's tumor type, location, tumor volume report/breast volume and overall support by the multidisciplinary team [5]. For years, conservative treatment was 
practiced on tumors whose diameter was less than $3 \mathrm{~cm}$.

The breast conserving therapy combines wide excision of the tumor (Figure 3 ), treatment of axillary lymph nodes and breast irradiation retained. It must meet three conditions: a similar survival to that achieved with mastectomy, a low risk of local recurrence and satisfactory cosmetic result [3] [6] .

The tumor was mostly localized in the upper outer quadrant (QSE), then the union QSE + QSI. This location is difficult to treat, in fact, the upper quadrant is the most uninhabited area of the breast, especially if the ptosis is important [7].

Gradually, new techniques have been used to fit each tumor localization (Figure 4), the breast size, shape and degree of breast ptosis [8]. The technical lipomodelling type has been used to improve breast deformities [9]. The round block also called mammoplasty by peri-areolar technique [8] has been the most common surgical technique (Figure 5 and Figure 6), consistent with the localization of lesions which was predominantly the upper outer quadrant (Figure 1). It is practiced for lesions near the areola of breast ptosis little, the technique is simple, reliable and fast realization [10]. The technique of the round block is one of the techniques that can be used also for tumors whose central location is rare.

Mammaplasty "inverted T" was the second most used in our study technique. The inverted $\mathrm{T}$ with superior pedicle is the first technique that was used Oncoplastic. It is suitable for breast ptosis and good volume, requiring skin desépidermisation keyhole, tumor resection in the lower quadrant union and balancing the contralateral breast [11]. Tumors of the external quadrants are treated with external technical. These tumors were frequent, the existence of a high concentration glandular within this area enables wide resection with easy remodeling [12]. The external technique permits us to remove the tumor and the skin facing off.

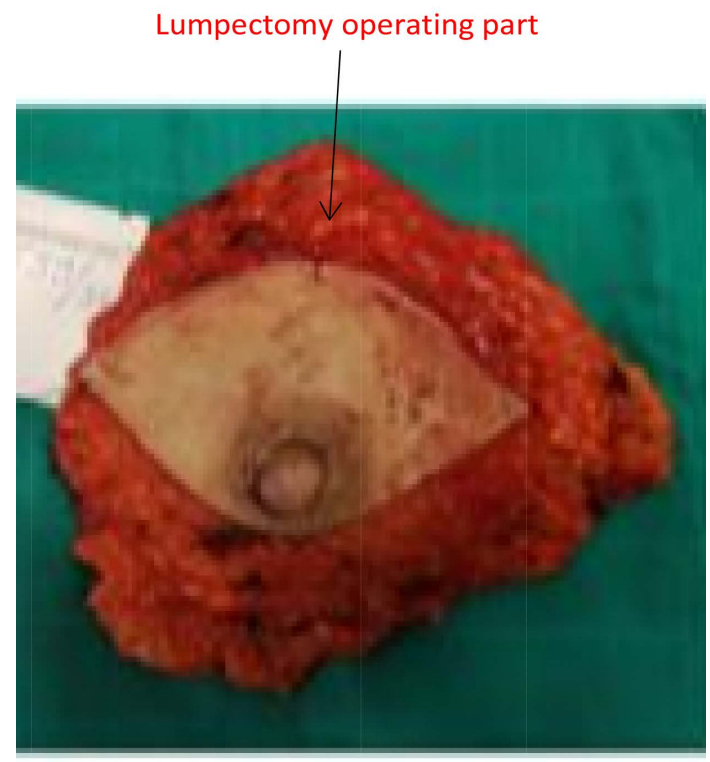

Figure 3. Wide excision of an ICC requires a gesture of Oncoplastic. 


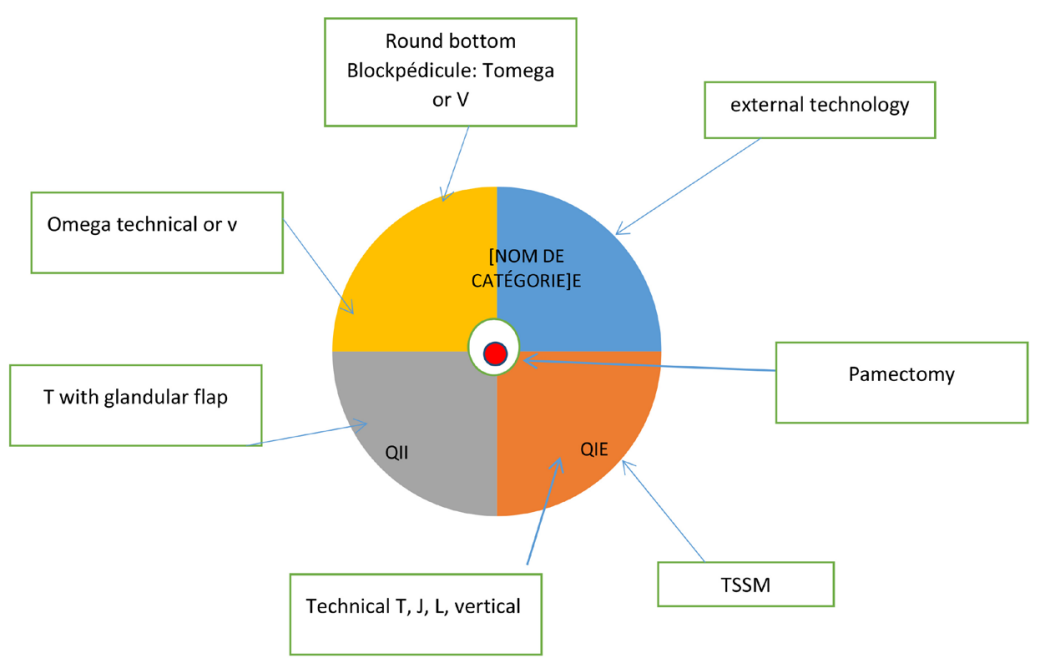

Figure 4. Schematic surgical techniques types according to the maps of the tumor.

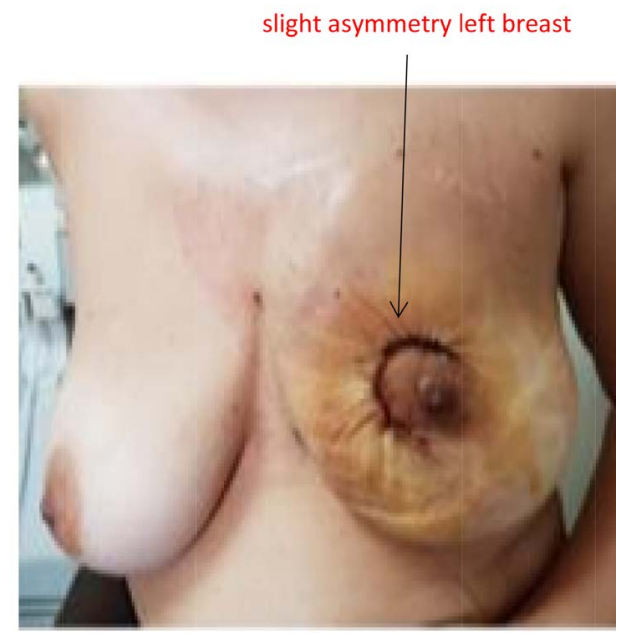

Figure 5. Oncoplastic by incision round block.

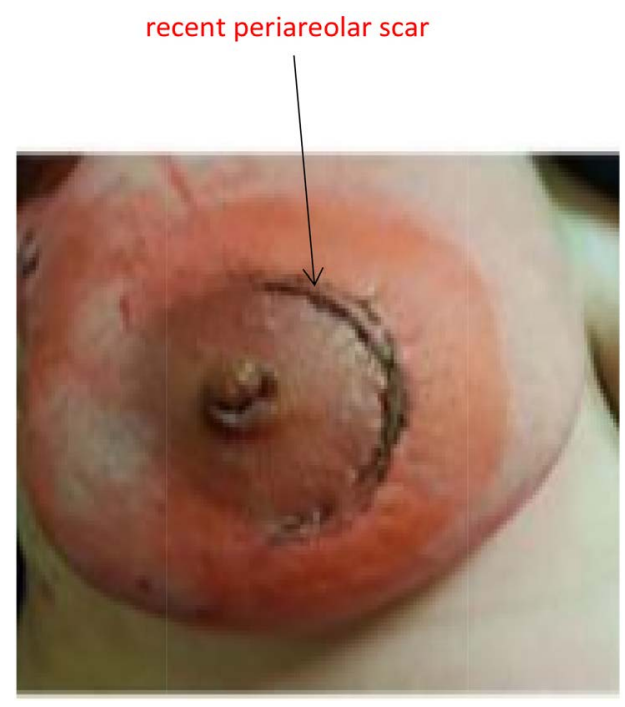

Figure 6. Oncoplastic by incision round block. 


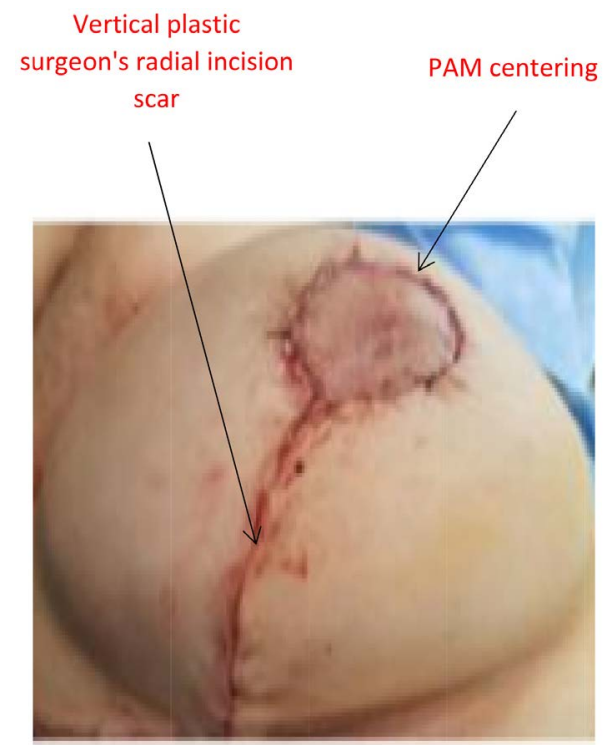

Figure 7. Excision of a lesion QII by radial incision and refocusing PAM.

post-operative image :

goodsymmetrization of the lying patient's breast

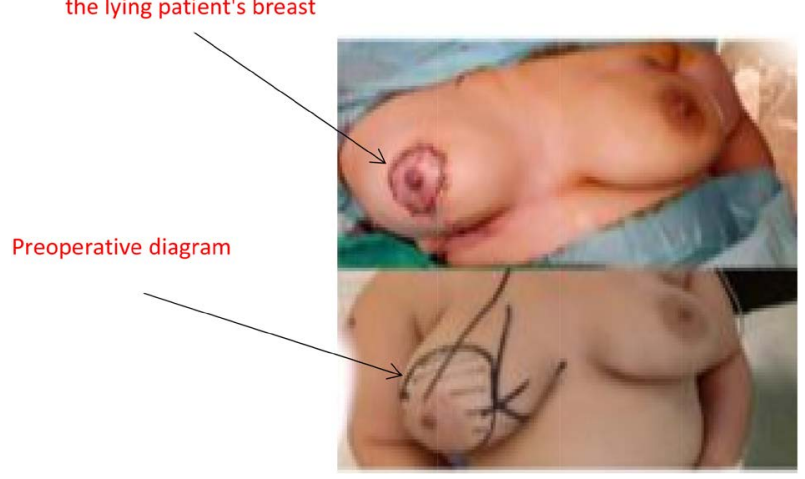

Figure 8. Balancing by breast reduction right breast.

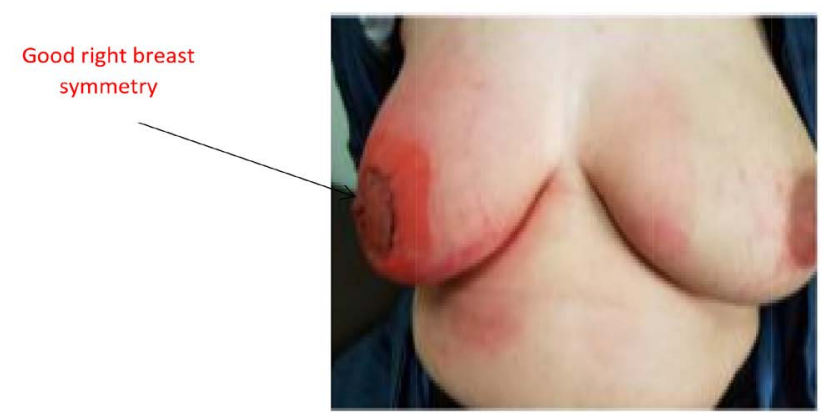

Figure 9. Aesthetic result after Oncoplastic.

For central tumors is carried Pamectomie, or removal of the nipple-breast plate (WFP) for tumors located within $2 \mathrm{~cm}$ of technical PAM.la Round block was extended to Pamectomie by some authors [10]. 
Table 1. Postoperative complications.

\begin{tabular}{ccc}
\hline & $\begin{array}{c}\text { our series } \\
\mathrm{N}=105 \%\end{array}$ & $\begin{array}{c}\text { Bouzoubaa W [13] } \\
\mathrm{N}=47 \%\end{array}$ \\
\hline immediate & 12 & 1 \\
lymphocele & 5 & 0 \\
lymphoedema & 0 & 10 \\
hematoma & 0 & 4 \\
necrosis & 0 & 8 \\
scar disunity & 0 & 2 \\
pains & 14 & 10 \\
retractile unsightly scar & 12 & 26 \\
breast asymmetry & 3 & 10 \\
cutaneous sclerosis & & \\
\hline
\end{tabular}

For tumors of the lower quadrant (Figures 7-9), the desépidermisation keyhole is performed, then either mammaplasty inverted $\mathrm{T}$ to superior pedicle, a pure vertical tuck, tuck or J or L. mammary furrow a specific technique: the technical sub-mammary fold (TSSM) with or without low back glandular.

The Oncoplastic, like all surgery is not free of complications (Table 1). Indeed, we noticed immediate and long-term complications. Immediate complications were lymphocele and lymphoedema.

The lymphocele and lymphedema [14] can be considered unpredictable surgical accidents, by cons in the literature hematoma, skin necrosis and disunity of reported scar, are predictable.

For late complications, there would be the risk factors of poor cosmetic results [13]: obesity, breast enlargement, the tumor sitting in the lower or central quadrants, the report tumor size/breast volume high/radiotherapy.

Some [13], the most important distortions are found to the central location tumors or lower include retractable clamps the axilla, deformations "eagle beak" and lumpectomy in the lower quadrants, the glandular defect, then the asymmetry of shape and breast volume.

\section{Conclusion}

Oncoplastic surgery is the gold standard for the treatment of invasive tumors less than $5 \mathrm{~cm}$, and non-inflammatory. In our structure, it ranges from simple to more elaborate remodeling techniques of mammoplasty and symmetrizations of the contralateral breast. These surgical choices are made accurately in consensual manner with the various stakeholders dealing with breast cancer at a multidisciplinary consultation meeting.

\section{Conflicts of Interest}

The authors declare no conflicts of interest regarding the publication of this paper. 


\section{References}

[1] Cotier-Save, I. and Rimarex, F. (2008) Principes Generals Oncoplastic Surgery. Annales within Esthétique. Plastic Surgery, 53, 102-111.

[2] Mauriac, L., et al. (1999) Chemotherapy for Operable Breast Carcinoma Larger than $3 \mathrm{~cm}$ : A Randomized Trial with UniCentre Has 124-Month Median Follow-Up. Annals of Oncology, 10, 47-52. https://doi.org/10.1023/A:1008337009350

[3] Diderot, V., Nnos, C. and Aucoutruer, J.S. (2004) Conservative Surgical Treatment of Breast Cancer. Cancer Radiotherapy, 8, 21-28. https://doi.org/10.1016/j.canrad.2003.10.008

[4] Cothier-Save, I. and Rimarex, F. (2008) General Principles of Oncoplastic Breast Surgery. Annals of Plastic Surgery Aesthetic, 53, 102-111.

[5] Cothier-Savey, I. and Rimareix, F. Principles of Oncoplastic Surgery and Immediate Breast Reconstruction after Mastectomy or Prophylactic Mastectomy for Carcinoma. Surgical Techniques. Plastic Reconstructive and Aesthetic Surgery.

[6] Jacob, J.A., Danforth, D.N., Cowan, K.H. D’angele, T., Steinberg, S.M., Pierce, L., et al. (1995) Ten Year Results of a Comparison of Conversations with Mastectomy in the Treatment of Stage of Talking with Mastectomy in the Treatment of Stage I and II Breast Cancer. The New England Journal of Medicine, 332, 907-911. https://doi.org/10.1056/NEJM199504063321402

[7] Malka, I., Villet, R., Fitoussi, A. and Salmon, R.J. (2010) Oncoplastic in the Conservative Treatment of Breast Cancer (Third Part). Techniques for Upper Quadrants. Journal of Visceral Surgery, 147, 453-460. https://doi.org/10.1016/j.jviscsurg.2010.08.017

[8] Fitoussi, A. (2010) Oncoplastic. Excerpts Updates for Gynecology and Obstetrics. CNGOF 34th National Days of 2010.

[9] Fitoussi, A.D., Berry, F., Fama, F., et al. (2010) Oncoplastic Breast Surgery for Cancer: Analysis of 540 Consecutive Cases (Articles Outcomes). Plastic and Reconstructive Surgery, 125, 454-462. https://doi.org/10.1097/PRS.0b013e3181c82d3e

[10] Body, G., Vuldamer, L. and Acker, O. (2010) Surgery Oncoplastic/Technique Round Block. CNGOF 35th National Days, Paris.

[11] Malka, I., Villet, R., Fitoussi, A. and Salmon, R.J. (2010) Oncoplastic in the Conservative Treatment of Breast Cancer (Part Two): Techniques for the Lower Quadrants. Journal of Visceral Surgery, 147, 378-388. https://doi.org/10.1016/j.jviscsurg.2010.08.011

[12] Fitoussi, A. (2017) Oncoplastic. Surgical Techniques in Gynecology. 2nd Edition, Elsevier, Amsterdam.

[13] Bouzoubaa, W. (2017) Conservative Treatment of Breast Cancer with Breast Oncoplastic. Dissertation Faculty of Medicine and Pharmacy in 2017 Fes.

[14] Wikipedia Dictionary. https://en:Wikipedia:org/wiki 